Research Article

\title{
Circulant Type Matrices with the Sum and Product of Fibonacci and Lucas Numbers
}

\author{
Zhaolin Jiang, ${ }^{1}$ Yanpeng Gong, ${ }^{2}$ and Yun Gao ${ }^{2}$ \\ ${ }^{1}$ Department of Mathematics, Linyi University, Linyi, Shandong 276000, China \\ ${ }^{2}$ Institute of Applied Mathematics, Shandong University of Technology, Zibo, Shandong 255049, China
}

Correspondence should be addressed to Zhaolin Jiang; jzh1208@sina.com

Received 28 April 2014; Accepted 4 June 2014; Published 19 June 2014

Academic Editor: Tongxing Li

Copyright (c) 2014 Zhaolin Jiang et al. This is an open access article distributed under the Creative Commons Attribution License, which permits unrestricted use, distribution, and reproduction in any medium, provided the original work is properly cited.

Circulant type matrices have become an important tool in solving differential equations. In this paper, we consider circulant type matrices, including the circulant and left circulant and $g$-circulant matrices with the sum and product of Fibonacci and Lucas numbers. Firstly, we discuss the invertibility of the circulant matrix and present the determinant and the inverse matrix by constructing the transformation matrices. Furthermore, the invertibility of the left circulant and $g$-circulant matrices is also discussed. We obtain the determinants and the inverse matrices of the left circulant and $g$-circulant matrices by utilizing the relation between left circulant, and $g$-circulant matrices and circulant matrix, respectively.

\section{Introduction}

Circulant matrices may play a crucial role for solving various differential equations. In [1], Ruiz-Claeyssen and dos Santos Leal introduced factor circulant matrices: matrices with the structure of circulants, but with the entries below the diagonal being multiplied by the same factor. The diagonalization of a circulant matrix and the spectral decomposition are conveniently generalized to block matrices with the structure of factor circulants. Matrix and partial differential equations involving factor circulants are considered. Wu and $\mathrm{Zou}$ in [2] discussed the existence and approximation of solutions of asymptotic or periodic boundary value problems of mixed functional differential equations. They focused on (5.13) in [2] with a circulant matrix, whose principal diagonal entries are zeroes. In [3], some Routh-Hurwitz stability conditions are generalized to the fractional order case. The authors considered the 1-system CML (10). They selected a circulant matrix, which reads a tridiagonal matrix. Ahmed and Elgazzar used coupled map lattices (CML) as an alternative approach to include spatial effects in fractional order systems (FOS). Consider the 1-system CML (10) in [4]. They claimed that the system is stable if all the eigenvalues of the circulant matrix satisfy (2) in [4]. Trench considered nonautonomous systems of linear differential equations (1) in [5] with some constraint on the coefficient matrix $A(t)$. One case is that $A(t)$ is a variable block circulant matrix. Kloeden et al. adopted the simplest approximation schemes for (1) in [6] with the Euler method, which reads (5) in [6]. They exploited that the covariance matrix of the increments can be embedded in a circulant matrix. The total loops can be done by fast Fourier transformation, which leads to a total computational cost of $O(m \log m)=O(n \log n)$. Guo et al. concerned on generic Dn-Hopf bifurcation to a delayed Hopfield-CohenGrossberg model of neural networks (5.17) in [7], where T denoted an interconnection matrix. They especially assumed $T$ is a symmetric circulant matrix. Lin and Yang discretized the partial integrodifferential equation (PIDE) in pricing options with the preconditioned conjugate gradient (PCG) method, which constructed the circulant preconditioners. By using FFT, the cost for each linear system is $O(n \log n)$, where $n$ is the size of the system in [8]. Lee et al. investigated a high-order compact (HOC) scheme for the general twodimensional (2D) linear partial differential equation (1.1) in [9] with a mixed derivative. Meanwhile, in order to establish the $2 \mathrm{D}$ combined compact difference (CCD2) scheme, they rewrote (1.1) in [9] into (2.1) in [9]. To write the CCD2 system in a concise style, they employed circulant matrix to obtain 
the corresponding whole CCD2 linear system (2.10) in [9], whose entries are circulant block.

Circulant type matrices have important applications in various disciplines including image processing, communications, signal processing, encoding, solving Toeplitz matrix problems, and least squares problems. They have been put on firm basis with the work of Davis [10], Jiang and Zhou [11], and Gray [12].

In [13], the authors pointed out the processes based on the eigenvalue of circulant type matrices with i.i.d. entries. There are discussions about the convergence in probability and in distribution of the spectral norm of circulant type matrices in [14]. The $g$-circulant matrices play an important role in various applications as well. For details, please refer to $[15,16]$ and the references therein. Ngondiep et al. showed the singular values of $g$-circulants in [17]. In $[18,19]$, the authors gave the limiting spectral distributions of left circulant matrices.

The Fibonacci and Lucas sequences are defined by the following recurrence relations $[20,21]$, respectively:

$$
\begin{gathered}
F_{n+2}=F_{n+1}+F_{n} \quad \text { where } F_{0}=0, F_{1}=1, \\
L_{n+2}=L_{n+1}+L_{n} \quad \text { where } L_{0}=2, L_{1}=1 .
\end{gathered}
$$

For $n \geq 0$, the first few values of the sequences are given by the following equation:

$$
\begin{array}{c|cccccccccc}
n & 0 & 1 & 2 & 3 & 4 & 5 & 6 & 7 & 8 & \cdots \\
\hline F_{n} & 0 & 1 & 1 & 2 & 3 & 5 & 8 & 13 & 21 & \cdots \\
L_{n} & 2 & 1 & 3 & 4 & 7 & 11 & 18 & 29 & 47 & \cdots
\end{array}
$$

Let $\alpha, \beta$ be the roots of characteristic equation $x^{2}-x-1=$ 0 ; then the Binet formulas of the sequences $\left\{F_{n}\right\}$ and $\left\{L_{n}\right\}$ have the form

$$
\begin{array}{cc}
F_{n}=\frac{\alpha^{n}-\beta^{n}}{\alpha-\beta}, & L_{n}=\alpha^{n}+\beta^{n}, \\
\alpha=\frac{1+\sqrt{5}}{2}, & \beta=\frac{1-\sqrt{5}}{2} .
\end{array}
$$

Let $\mathscr{F}_{n}=F_{n} \cdot L_{n}$ and $\mathscr{L}_{n}=F_{n}+L_{n}$, so we can get two new sequences $\mathscr{F}_{n}$ and $\mathscr{L}_{n}$ [22]. The two sequences are defined by the following recurrence relations, respectively:

$$
\begin{gathered}
\mathscr{F}_{n+2}=3 \mathscr{F}_{n+1}+\mathscr{F}_{n}, \quad \text { where } \mathscr{F}_{0}=0, \mathscr{F}_{1}=1, \\
\mathscr{L}_{n+2}=\mathscr{L}_{n+1}+\mathscr{L}_{n}, \quad \text { where } \mathscr{L}_{0}=2, \mathscr{L}_{1}=2 .
\end{gathered}
$$

For $n \geq 0$, the first few values of the sequences are given by the following equation:

$$
\begin{array}{c|cccccccccc}
n & 0 & 1 & 2 & 3 & 4 & 5 & 6 & 7 & 8 & \cdots \\
\hline \mathscr{F}_{n} & 0 & 1 & 3 & 8 & 21 & 55 & 144 & 377 & 987 & \cdots \\
\mathscr{L}_{n} & 2 & 2 & 4 & 6 & 10 & 16 & 26 & 42 & 68 & \cdots
\end{array}
$$

The $\mathscr{F}_{n}$ is given by the formula $\mathscr{F}_{n}=\left(\alpha_{1}^{n}-\beta_{1}^{n}\right) /\left(\alpha_{1}-\beta_{1}\right)$, where $\alpha_{1}, \beta_{1}$ are the roots of $x^{2}-3 x+1=0 . \mathscr{L}_{n}$ is given by the formula $\mathscr{L}_{n}=F_{n}+L_{n}=\left(\alpha^{n}-\beta^{n}\right) /(\alpha-\beta)+\left(\alpha^{n}+\beta^{n}\right)$, where $\alpha, \beta$ are the roots of $x^{2}-x-1=0$.

Besides, some scholars have given various algorithms for the determinants and inverses of nonsingular circulant matrices $[10,11]$. Unfortunately, the computational complexities of these algorithms are very amazing with the order of matrix increasing. However, some authors gave the explicit determinants and inverse of circulant and skewcirculant involving Fibonacci and Lucas numbers. For example, Dazheng gave the determinant of the Fibonacci-Lucas quasicyclic matrices in [20]. Shen et al. considered circulant matrices with Fibonacci and Lucas numbers and presented their explicit determinants and inverses by constructing the transformation matrices [21]. Jaiswal evaluated some determinants of circulant whose elements are the generalized Fibonacci numbers [23]. Lind presented the determinants of circulant and skew-circulant involving Fibonacci numbers [24]. Bozkurt and Tam gave determinants and inverses of circulant matrices with Jacobsthal and Jacobsthal-Lucas numbers [25].

In [22], the authors gave some determinantal and permanental representations of $\mathscr{F}_{n}$ and $\mathscr{L}_{n}$ and complex factorization formulas. The purpose of this paper is to obtain the explicit determinants and inverse of circulant type matrices by some perfect properties of $\mathscr{F}_{n}$ and $\mathscr{L}_{n}$.

In this paper, we adopt the following two conventions $0^{0}=1$, and for any sequence $\left\{a_{n}\right\}, \sum_{k=i}^{n} a_{k}=0$ in the case $i>n$.

Definition 1 (see $[10,11]$ ). In a circulant matrix (or right circulant matrix [26])

$$
\operatorname{Circ}\left(a_{1}, a_{2}, \ldots, a_{n}\right)=\left[\begin{array}{cccc}
a_{1} & a_{2} & \cdots & a_{n} \\
a_{n} & a_{1} & \cdots & a_{n-1} \\
\vdots & \vdots & & \vdots \\
a_{2} & a_{3} & \cdots & a_{1}
\end{array}\right],
$$

each row is a cyclic shift of the row above to the right.

Circulant matrix is a special case of a Toeplitz matrix. It is evidently determined by its first row (or column).

Definition 2 (see $[11,26]$ ). In a left circulant matrix (or reverse circulant matrix $[13,14,18,19])$

$$
\operatorname{LCirc}\left(a_{1}, a_{2}, \ldots, a_{n}\right)=\left[\begin{array}{cccc}
a_{1} & a_{2} & \cdots & a_{n} \\
a_{2} & a_{3} & \cdots & a_{1} \\
\vdots & \vdots & & \vdots \\
a_{n} & a_{1} & \cdots & a_{n-1}
\end{array}\right],
$$

each row is a cyclic shift of the row above to the left.

Left circulant matrix is a special Hankel matrix.

Definition 3 (see $[14,27]$ ). A $g$-circulant matrix is an $n \times n$ complex matrix with the following form:

$$
A_{g, n}=\left(\begin{array}{cccc}
a_{1} & a_{2} & \cdots & a_{n} \\
a_{n-g+1} & a_{n-g+2} & \cdots & a_{n-g} \\
a_{n-2 g+1} & a_{n-2 g+2} & \cdots & a_{n-2 g} \\
\vdots & \vdots & \ddots & \vdots \\
a_{g+1} & a_{g+2} & \cdots & a_{g}
\end{array}\right) \text {, }
$$


where $g$ is a nonnegative integer and each of the subscripts is understood to be reduced modulo $n$.

The first row of $A_{g, n}$ is $\left(a_{1}, a_{2}, \ldots, a_{n}\right)$; its $(j+1)$ th row is obtained by giving its $j$ th row a right circular shift by $g$ positions (equivalently, $g \bmod n$ positions). Note that $g=1$ or $g=n+1$ yields the standard circulant matrix. If $g=n-1$, then we obtain the left circulant matrix.

Lemma 4 (see [21]). Let $A=\operatorname{Circ}\left(a_{1}, a_{2}, \ldots, a_{n}\right)$ be a circulant matrix; then one has

(i) $A$ is invertible if and only if $f\left(\omega^{k}\right) \neq 0,(k=0,1$, $2, \ldots, n-1)$, where $f(x)=\sum_{j=1}^{n} a_{j} x^{j-1}$ and $\omega=$ $\exp (2 \pi i / n)$;

(ii) If $A$ is invertible, then the inverse $A^{-1}$ of $A$ is a circulant matrix.

Lemma 5. Define

$$
\Delta:=\left(\begin{array}{cccccc}
1 & 0 & 0 & \cdots & 0 & 0 \\
0 & 0 & 0 & \cdots & 0 & 1 \\
0 & 0 & 0 & \cdots & 1 & 0 \\
\vdots & \vdots & \ddots & & \vdots & \\
0 & 0 & 1 & \cdots & 0 & 0 \\
0 & 1 & 0 & \cdots & 0 & 0
\end{array}\right)
$$

the matrix $\Delta$ is an orthogonal cyclic shift matrix (and a left circulant matrix). It holds that $\operatorname{LCirc}\left(a_{1}, a_{2}, \ldots, a_{n}\right)=$ $\Delta \operatorname{Circ}\left(a_{1}, a_{2}, \ldots, a_{n}\right)$.

Lemma 6 (see [27]). The $n \times n$ matrix $\mathbb{Q}_{g}$ is unitary if and only if $(n, g)=1$, where $\mathbb{Q}_{g}$ is a $g$-circulant matrix with the first row $e^{*}=[1,0, \ldots, 0]$.

Lemma 7 (see [27]). $A_{g, n}$ is a g-circulant matrix with the first row $\left[a_{1}, a_{2}, \ldots, a_{n}\right]$ if and only if $A_{g, n}=\mathbb{Q}_{g} C$, where $C=$ $\operatorname{Circ}\left(a_{1}, a_{2}, \ldots, a_{n}\right)$.

\section{Determinant and Inverse of a Circulant Matrix with the Product of the Fibonacci and Lucas Numbers}

In this section, let $\mathscr{A}_{n}=\operatorname{Circ}\left(\mathscr{F}_{1}, \mathscr{F}_{2}, \ldots, \mathscr{F}_{n}\right)$ be a circulant matrix. Firstly, we give the determinant equation of matrix $\mathscr{A}_{n}$. Afterwards, we prove that $\mathscr{A}_{n}$ is an invertible matrix for $n>2$, and then we find the inverse of the matrix $\mathscr{A}_{n}$.

Theorem 8. Let $\mathscr{A}_{n}=\operatorname{Circ}\left(\mathscr{F}_{1}, \mathscr{F}_{2}, \ldots, \mathscr{F}_{n}\right)$ be a circulant matrix; then one has

$$
\begin{aligned}
\operatorname{det} \mathscr{A}_{n}= & \left(1-\mathscr{F}_{n+1}\right)^{n-1} \\
& +\left(-\mathscr{F}_{n}\right)^{n-2} \sum_{k=1}^{n-1}\left(-\mathscr{F}_{k}\right)\left(\frac{1-\mathscr{F}_{n+1}}{-\mathscr{F}_{n}}\right)^{k-1},
\end{aligned}
$$

where $\mathscr{F}_{n}$ is the nth $F_{n} \cdot L_{n}$ number.
Proof. Obviously, det $\mathscr{A}_{1}=1$ satisfies (10). In the case $n>1$, let

$$
\Gamma=\left(\begin{array}{ccccccccc}
1 & & & & & & \\
-3 & & & & & & 1 \\
1 & & & & & 1 & -3 \\
0 & & 0 & & 1 & -3 & 1 \\
\vdots & & & & \ddots & . & \\
0 & & & 1 & \ddots & \ddots & & \\
0 & & 1 & -3 & \ddots & & 0 & \\
0 & 1 & -3 & 1 & & & &
\end{array}\right)_{n \times n},
$$

$$
\Pi_{1}=\left(\begin{array}{cccccc}
1 & 0 & 0 & \cdots & 0 & 0 \\
0 & \left(\frac{-\mathscr{F}_{n}}{\mathscr{F}_{1}-\mathscr{F}_{n+1}}\right)^{n-2} & 0 & \cdots & 0 & 1 \\
0 & \left(\frac{-\mathscr{F}_{n}}{\mathscr{F}_{1}-\mathscr{F}_{n+1}}\right)^{n-3} & 0 & \cdots & 1 & 0 \\
\vdots & \vdots & \vdots & \ddots & \vdots & \vdots \\
0 & \frac{-\mathscr{F}_{n}}{\mathscr{F}_{1}-\mathscr{F}_{n+1}} & 1 & \cdots & 0 & 0 \\
0 & 1 & 0 & \cdots & 0 & 0
\end{array}\right)_{n \times n}
$$

We can obtain

$\Gamma \mathscr{A}_{n} \Pi_{1}$

$$
=\left(\begin{array}{cccccc}
\mathscr{F}_{1} & f_{n}^{\prime} & \mathscr{F}_{n-1} & \mathscr{F}_{n-2} & \ldots & \mathscr{F}_{2} \\
0 & f_{n} & -\mathscr{F}_{n-2} & -\mathscr{F}_{n-3} & \cdots & -\mathscr{F}_{1} \\
0 & 0 & \mathscr{F}_{1}-\mathscr{F}_{n+1} & & & \\
0 & 0 & \mathscr{F}_{n} & \mathscr{F}_{1}-\mathscr{F}_{n+1} & & \\
\vdots & \vdots & & \mathscr{F}_{n} & & \\
0 & 0 & & 0 & \mathscr{F}_{1}-\mathscr{F}_{n+1}
\end{array}\right) \text {, }
$$

where

$$
\begin{gathered}
f_{n}=\mathscr{F}_{1}-3 \mathscr{F}_{n}+\sum_{k=1}^{n-2}\left(-\mathscr{F}_{k}\right)\left(\frac{-\mathscr{F}_{n}}{\mathscr{F}_{1}-\mathscr{F}_{n+1}}\right)^{n-(k+1)}, \\
f_{n}^{\prime}=\sum_{k=1}^{n-1} \mathscr{F}_{k+1}\left(\frac{-\mathscr{F}_{n}}{\mathscr{F}_{1}-\mathscr{F}_{n+1}}\right)^{n-(k+1)} .
\end{gathered}
$$

We obtain

$\operatorname{det} \Gamma \operatorname{det} \mathscr{A}_{n} \operatorname{det} \Pi_{1}$

$$
\begin{aligned}
= & \mathscr{F}_{1}\left[\mathscr{F}_{1}-3 \mathscr{F}_{n}+\sum_{k=1}^{n-2}\left(-\mathscr{F}_{k}\right)\left(\frac{-\mathscr{F}_{n}}{\mathscr{F}_{1}-\mathscr{F}_{n+1}}\right)^{n-(k+1)}\right] \\
& \times\left(\mathscr{F}_{1}-\mathscr{F}_{n+1}\right)^{n-2} \\
= & \mathscr{F}_{1}\left[\mathscr{F}_{1}-\mathscr{F}_{n+1}+\sum_{k=1}^{n-1}\left(-\mathscr{F}_{k}\right)\left(\frac{-\mathscr{F}_{n}}{\mathscr{F}_{1}-\mathscr{F}_{n+1}}\right)^{n-(k+1)}\right]
\end{aligned}
$$




$$
\begin{aligned}
& \times\left(\mathscr{F}_{1}-\mathscr{F}_{n+1}\right)^{n-2} \\
= & \left(1-\mathscr{F}_{n+1}\right)^{n-1} \\
& +\left(-\mathscr{F}_{n}\right)^{n-2} \sum_{k=1}^{n-1}\left(-\mathscr{F}_{k}\right)\left(\frac{1-\mathscr{F}_{n+1}}{-\mathscr{F}_{n}}\right)^{k-1},
\end{aligned}
$$

while

$$
\operatorname{det} \Gamma=\operatorname{det} \Pi_{1}=(-1)^{(n-1)(n-2) / 2},
$$

we have

$$
\begin{aligned}
\operatorname{det} \mathscr{A}_{n}= & \left(1-\mathscr{F}_{n+1}\right)^{n-1} \\
& +\left(-\mathscr{F}_{n}\right)^{n-2} \sum_{k=1}^{n-1}\left(-\mathscr{F}_{k}\right)\left(\frac{1-\mathscr{F}_{n+1}}{-\mathscr{F}_{n}}\right)^{k-1} .
\end{aligned}
$$

Thus, the proof is completed.

Theorem 9. Let $\mathscr{A}_{n}=\operatorname{Circ}\left(\mathscr{F}_{1}, \mathscr{F}_{2}, \ldots, \mathscr{F}_{n}\right)$ be a circulant matrix; if $n>2$, then $\mathscr{A}_{n}$ is an invertible matrix.

Proof. When $n=3$, in Theorem 8, we have $\operatorname{det} \mathscr{A}_{3}=468 \neq 0$; hence $\mathscr{A}_{3}$ is invertible. In the case $n>3$, since $\mathscr{F}_{n}=\left(\alpha_{1}^{n}-\beta_{1}^{n}\right) /$ $\left(\alpha_{1}-\beta_{1}\right)$, where $\alpha_{1}+\beta_{1}=3, \alpha_{1} \cdot \beta_{1}=1$. We have

$$
\begin{aligned}
f\left(\omega^{k}\right)= & \sum_{j=1}^{n} \mathscr{F}_{j}\left(\omega^{k}\right)^{j-1} \\
= & \frac{1}{\alpha_{1}-\beta_{1}} \sum_{j=1}^{n}\left(\alpha_{1}^{j}-\beta_{1}^{j}\right)\left(\omega^{k}\right)^{j-1} \\
= & \frac{1}{\alpha_{1}-\beta_{1}}\left[\frac{\alpha_{1}\left(1-\alpha_{1}^{n}\right)}{1-\alpha_{1} \omega^{k}}-\frac{\beta_{1}\left(1-\beta_{1}^{n}\right)}{1-\beta_{1} \omega^{k}}\right] \\
= & \frac{1}{\alpha_{1}-\beta_{1}}\left[\frac{\left(\alpha_{1}-\beta_{1}\right)-\left(\alpha_{1}^{n+1}-\beta_{1}^{n+1}\right)}{1-\left(\alpha_{1}+\beta_{1}\right) \omega^{k}+\alpha_{1} \beta_{1} \omega^{2 k}}\right] \\
& +\frac{1}{\alpha_{1}-\beta_{1}}\left[\frac{\alpha_{1} \beta_{1}\left(\alpha_{1}^{n}-\beta_{1}^{n}\right) \omega^{k}}{1-\left(\alpha_{1}+\beta_{1}\right) \omega^{k}+\alpha_{1} \beta_{1} \omega^{2 k}}\right] \\
= & \frac{1-\mathscr{F}_{n+1}+\mathscr{F}_{n} \omega^{k}}{1-3 \omega^{k}+\omega^{2 k}} \quad(k=1,2, \ldots, n-1) .
\end{aligned}
$$

If there exists $\omega^{l}(l=1,2, \ldots, n-1)$ such that $f\left(\omega^{l}\right)=0$, we obtain $1-\mathscr{F}_{n+1}+\mathscr{F}_{n} \omega^{l}=0$ for $1-3 \omega^{l}+\omega^{2 l} \neq 0$; thus, $\omega^{l}=$ $\left(\mathscr{F}_{n+1}-1\right) / \mathscr{F}_{n}$ is a real number. While $\omega^{l}=\exp (2 l \pi i / n)=$ $\cos (2 l \pi / n)+i \sin (2 l \pi / n)$, hence, $\sin (2 l \pi / n)=0$, so we have $\omega^{l}=-1$ for $0<2 l \pi / n<2 \pi$. But $x=-1$ is not the root of equation $1-\mathscr{F}_{n+1}+\mathscr{F}_{n} x=0(n>3)$. We obtain $f\left(\omega^{k}\right) \neq 0$ for any $\omega^{k}(k=1,2, \ldots, n-1)$, while $f(1)=\sum_{j=1}^{n} \mathscr{F}_{j}=$ $\mathscr{F}_{n+1}-\mathscr{F}_{n}-1 \neq 0$. By Lemma 4 , the proof is completed.
Lemma 10. Let the matrix $\mathscr{G}=\left[g_{i, j}\right]_{i, j=1}^{n-2}$ be of the form

$$
g_{i j}= \begin{cases}\mathscr{F}_{1}-\mathscr{F}_{n+1}, & i=j, \\ \mathscr{F}_{n}, & i=j+1, \\ 0, & \text { otherwise, }\end{cases}
$$

and then the inverse $\mathscr{G}^{-1}=\left[g_{i, j}^{\prime}\right]_{i, j=1}^{n-2}$ of the matrix $\mathscr{G}$ is equal to

$$
g_{i, j}^{\prime}= \begin{cases}\frac{\left(-\mathscr{F}_{n}\right)^{i-j}}{\left(\mathscr{F}_{1}-\mathscr{F}_{n+1}\right)^{i-j+1}}, & i \geq j, \\ 0, & i<j .\end{cases}
$$

Proof. Let $c_{i j}=\sum_{k=1}^{n-2} g_{i k} g_{k j}^{\prime}$. Obviously, $c_{i, j}=0$ for $i<j$. In the case $i=j$, we obtain $c_{i i}=g_{i i} g_{i i}^{\prime}=\left(\mathscr{F}_{1}-\mathscr{F}_{n+1}\right) \cdot\left(1 /\left(\mathscr{F}_{1}-\right.\right.$ $\left.\left.\mathscr{F}_{n+1}\right)\right)=1$. For $i \geq j+1$, we obtain

$$
\begin{aligned}
c_{i j}= & \sum_{k=1}^{n-2} g_{i k} g_{k j}^{\prime}=g_{i, i-1} g_{i-1, j}^{\prime}+g_{i, i} g_{i, j}^{\prime} \\
= & \mathscr{F}_{n} \cdot \frac{\left(-\mathscr{F}_{n}\right)^{i-j-1}}{\left(\mathscr{F}_{1}-\mathscr{F}_{n+1}\right)^{i-j}} \\
& +\left(\mathscr{F}_{1}-\mathscr{F}_{n+1}\right) \cdot \frac{\left(-\mathscr{F}_{n}\right)^{i-j}}{\left(\mathscr{F}_{1}-\mathscr{F}_{n+1}\right)^{i-j+1}}=0 .
\end{aligned}
$$

We verify $\mathscr{G} \mathscr{G}^{-1}=I_{n-2}$, where $I_{n-2}$ is the $(n-2) \times(n-2)$ identity matrix. Similarly, we can verify $\mathscr{G}^{-1} \mathscr{G}=I_{n-2}$. Thus, the proof is completed.

Theorem 11. Let $\mathscr{A}_{n}=\operatorname{Circ}\left(\mathscr{F}_{1}, \mathscr{F}_{2}, \ldots, \mathscr{F}_{n}\right)(n>2)$ be a circulant matrix; then one has

$$
\begin{aligned}
\mathscr{A}_{n}^{-1}=\frac{1}{f_{n}} \operatorname{Circ}(1 & -\sum_{i=1}^{n-2} \frac{\mathscr{F}_{n-i}\left(-\mathscr{F}_{n}\right)^{i-1}}{\left(\mathscr{F}_{1}-\mathscr{F}_{n+1}\right)^{i}}, \\
& -3+\sum_{i=1}^{n-2} \frac{\mathscr{F}_{n-1-i}\left(-\mathscr{F}_{n}\right)^{i-1}}{\left(\mathscr{F}_{1}-\mathscr{F}_{n+1}\right)^{i}}, \frac{1}{\mathscr{F}_{1}-\mathscr{F}_{n+1}}, \\
& \frac{-\mathscr{F}_{n}}{\left(\mathscr{F}_{1}-\mathscr{F}_{n+1}\right)^{2}}, \frac{\left(-\mathscr{F}_{n}\right)^{2}}{\left(\mathscr{F}_{1}-\mathscr{F}_{n+1}\right)^{3}}, \ldots, \\
& \left.\frac{\left(-\mathscr{F}_{n}\right)^{n-3}}{\left(\mathscr{F}_{1}-\mathscr{F}_{n+1}\right)^{n-2}}\right)
\end{aligned}
$$

where

$$
f_{n}=\mathscr{F}_{1}-3 \mathscr{F}_{n}+\sum_{k=1}^{n-2}\left(-\mathscr{F}_{k}\right)\left(\frac{-\mathscr{F}_{n}}{\mathscr{F}_{1}-\mathscr{F}_{n+1}}\right)^{n-(k+1)}
$$


Proof. Let

$$
\Pi_{2}=\left(\begin{array}{ccccc}
1 & -f_{n}^{\prime} & -\frac{f_{n}^{\prime}}{f_{n}} \mathscr{F}_{n-2}-\mathscr{F}_{n-1} & \cdots & -\frac{f_{n}^{\prime}}{f_{n}} \mathscr{F}_{1} \\
0 & 1 & \frac{\mathscr{F}_{n-2}}{f_{n}} & \cdots & \frac{\mathscr{F}_{1}}{f_{n}} \\
0 & 0 & 1 & \cdots & 0 \\
\vdots & \vdots & \vdots & \ddots & \vdots \\
0 & 0 & 0 & \cdots & 1
\end{array}\right),
$$

where

$$
\begin{gathered}
f_{n}=\mathscr{F}_{1}-3 \mathscr{F}_{n}+\sum_{k=1}^{n-2}\left(-\mathscr{F}_{k}\right)\left(\frac{-\mathscr{F}_{n}}{\mathscr{F}_{1}-\mathscr{F}_{n+1}}\right)^{n-(k+1)}, \\
f_{n}^{\prime}=\sum_{k=1}^{n-1} \mathscr{F}_{k+1}\left(\frac{-\mathscr{F}_{n}}{\mathscr{F}_{1}-\mathscr{F}_{n+1}}\right)^{n-(k+1)} .
\end{gathered}
$$

We have

$$
\Gamma \mathscr{A}_{n} \Pi_{1} \Pi_{2}=\mathscr{D}_{1} \oplus \mathscr{G}
$$

where $\mathscr{D}_{1}=\operatorname{diag}\left(\mathscr{F}_{1}, f_{n}\right)$ is a diagonal matrix and $\mathscr{D}_{1} \oplus \mathscr{G}$ is the direct sum of $\mathscr{D}_{1}$ and $\mathscr{G}$. If we denote $\Pi=\Pi_{1} \Pi_{2}$, then we obtain

$$
\mathscr{A}_{n}^{-1}=\Pi\left(\mathscr{D}_{1}^{-1} \oplus \mathscr{G}^{-1}\right) \Gamma
$$

Since the last row elements of the matrix $\Pi$ are

$$
0,1, \frac{\mathscr{F}_{n-2}}{f_{n}}, \frac{\mathscr{F}_{n-3}}{f_{n}}, \ldots, \frac{\mathscr{F}_{2}}{f_{n}}, \frac{\mathscr{F}_{1}}{f_{n}}
$$

By Lemma 10, if we let $\mathscr{A}_{n}^{-1}=\operatorname{Circ}\left(x_{1}, x_{2}, \ldots, x_{n}\right)$, its last row elements are given by the following equations:

$$
\begin{aligned}
x_{2}= & -\frac{3}{f_{n}}+\frac{1}{f_{n}} \sum_{i=1}^{n-2} \frac{\mathscr{F}_{n-1-i}\left(-\mathscr{F}_{n}\right)^{i-1}}{\left(\mathscr{F}_{1}-\mathscr{F}_{n+1}\right)^{i}}, \\
x_{3}= & \frac{\mathscr{F}_{1}}{f_{n}\left(\mathscr{F}_{1}-\mathscr{F}_{n+1}\right)}, \\
x_{4}= & \frac{1}{f_{n}} \sum_{i=1}^{2} \frac{\mathscr{F}_{3-i}\left(-\mathscr{F}_{n}\right)^{i-1}}{\left(\mathscr{F}_{1}-\mathscr{F}_{n+1}\right)^{i}}-\frac{3 \mathscr{F}_{1}}{f_{n}\left(\mathscr{F}_{1}-\mathscr{F}_{n+1}\right)}, \\
x_{5}= & \frac{1}{f_{n}} \sum_{i=1}^{3} \frac{\mathscr{F}_{4-i}\left(-\mathscr{F}_{n}\right)^{i-1}}{\left(\mathscr{F}_{1}-\mathscr{F}_{n+1}\right)^{i}}-\frac{3}{f_{n}} \sum_{i=1}^{2} \frac{\mathscr{F}_{3-i}\left(-\mathscr{F}_{n}\right)^{i-1}}{\left(\mathscr{F}_{1}-\mathscr{F}_{n+1}\right)^{i}} \\
& +\frac{\mathscr{F}_{1}}{f_{n}\left(\mathscr{F}_{1}-\mathscr{F}_{n+1}\right)},
\end{aligned}
$$

$$
\begin{aligned}
x_{n}= & \frac{1}{f_{n}} \sum_{i=1}^{n-2} \frac{\mathscr{F}_{n-1-i}\left(-\mathscr{F}_{n}\right)^{i-1}}{\left(\mathscr{F}_{1}-\mathscr{F}_{n+1}\right)^{i}}-\frac{3}{f_{n}} \sum_{i=1}^{n-3} \frac{\mathscr{F}_{n-2-i}\left(-\mathscr{F}_{n}\right)^{i-1}}{\left(\mathscr{F}_{1}-\mathscr{F}_{n+1}\right)^{i}} \\
& +\frac{1}{f_{n}} \sum_{i=1}^{n-4} \frac{\mathscr{F}_{n-3-i}\left(-\mathscr{F}_{n}\right)^{i-1}}{\left(\mathscr{F}_{1}-\mathscr{F}_{n+1}\right)^{i}}, \\
x_{1}= & \frac{1}{f_{n}}-\frac{3}{f_{n}} \sum_{i=1}^{n-2} \frac{\mathscr{F}_{n-1-i}\left(-\mathscr{F}_{n}\right)^{i-1}}{\left(\mathscr{F}_{1}-\mathscr{F}_{n+1}\right)^{i}} \\
& +\frac{1}{f_{n}} \sum_{i=1}^{n-3} \frac{\mathscr{F}_{n-2-i}\left(-\mathscr{F}_{n}\right)^{i-1}}{\left(\mathscr{F}_{1}-\mathscr{F}_{n+1}\right)^{i} .}
\end{aligned}
$$

Let $C_{n}^{(j)}=\sum_{i=1}^{j}\left(\mathscr{F}_{j+1-i}\left(-\mathscr{F}_{n}\right)^{i-1} /\left(\mathscr{F}_{1}-\mathscr{F}_{n+1}\right)^{i}\right)(j=1$, $2, \ldots, n-2)$; we have

$$
C_{n}^{(2)}-3 C_{n}^{(1)}
$$

$$
\begin{aligned}
& =-\frac{3 \mathscr{F}_{1}}{\mathscr{F}_{1}-\mathscr{F}_{n+1}}+\sum_{i=1}^{2} \frac{\mathscr{F}_{3-i}\left(-\mathscr{F}_{n}\right)^{i-1}}{\left(\mathscr{F}_{1}-\mathscr{F}_{n+1}\right)^{i}} \\
& =\frac{-\mathscr{F}_{n}}{\left(\mathscr{F}_{1}-\mathscr{F}_{n+1}\right)^{2}}, \\
& -3 C_{n}^{(n-2)}+C_{n}^{(n-3)} \\
& =-3 \sum_{i=1}^{n-2} \frac{\mathscr{F}_{n-1-i}\left(-\mathscr{F}_{n}\right)^{i-1}}{\left(\mathscr{F}_{1}-\mathscr{F}_{n+1}\right)^{i}}+\sum_{i=1}^{n-3} \frac{\mathscr{F}_{n-i-2}\left(-\mathscr{F}_{n}\right)^{i-1}}{\left(\mathscr{F}_{1}-\mathscr{F}_{n+1}\right)^{i}} \\
& =\frac{\left(-3 \mathscr{F}_{1}\right)\left(-\mathscr{F}_{n}\right)^{n-3}}{\left(\mathscr{F}_{1}-\mathscr{F}_{n+1}\right)^{n-2}}+\sum_{i=1}^{n-3} \frac{-\mathscr{F}_{n-i}\left(-\mathscr{F}_{n}\right)^{i-1}}{\left(\mathscr{F}_{1}-\mathscr{F}_{n+1}\right)^{i}} \\
& =\sum_{i=1}^{n-2} \frac{-\mathscr{F}_{n-i}\left(-\mathscr{F}_{n}\right)^{i-1}}{\left(\mathscr{F}_{1}-\mathscr{F}_{n+1}\right)^{i}} \\
& C_{n}^{(j+2)}-3 C_{n}^{(j+1)}+C_{n}^{(j)} \\
& =\sum_{i=1}^{j+2} \frac{\mathscr{F}_{j+3-i}\left(-\mathscr{F}_{n}\right)^{i-1}}{\left(\mathscr{F}_{1}-\mathscr{F}_{n+1}\right)^{i}}-3 \sum_{i=1}^{j+1} \frac{\mathscr{F}_{j+2-i}\left(-\mathscr{F}_{n}\right)^{i-1}}{\left(\mathscr{F}_{1}-\mathscr{F}_{n+1}\right)^{i}} \\
& +\sum_{i=1}^{j} \frac{\mathscr{F}_{j+1-i}\left(-\mathscr{F}_{n}\right)^{i-1}}{\left(\mathscr{F}_{1}-\mathscr{F}_{n+1}\right)^{i}} \\
& =\frac{\mathscr{F}_{2}\left(-\mathscr{F}_{n}\right)^{j}}{\left(\mathscr{F}_{1}-\mathscr{F}_{n+1}\right)^{j+1}}+\frac{\mathscr{F}_{1}\left(-\mathscr{F}_{n}\right)^{j+1}}{\left(\mathscr{F}_{1}-\mathscr{F}_{n+1}\right)^{j+2}} \\
& -\frac{3 \mathscr{F}_{1}\left(-\mathscr{F}_{n}\right)^{j}}{\left(\mathscr{F}_{1}-\mathscr{F}_{n+1}\right)^{j+1}}
\end{aligned}
$$




$$
\begin{aligned}
& +\sum_{i=1}^{j} \frac{\left(\mathscr{F}_{j+3-i}-3 \mathscr{F}_{j+2-i}+\mathscr{F}_{j+1-i}\right)\left(-\mathscr{F}_{n}\right)^{i-1}}{\left(\mathscr{F}_{1}-\mathscr{F}_{n+1}\right)^{j+1}} \\
= & \frac{\left(-\mathscr{F}_{n}\right)^{j+1}}{\left(\mathscr{F}_{1}-\mathscr{F}_{n+1}\right)^{j+2}} \quad(j=1,2, \ldots, n-4) .
\end{aligned}
$$

We obtain

$$
\begin{aligned}
& \mathscr{A}_{n}^{-1}=\operatorname{Circ}\left(\frac{1-3 C_{n}^{(n-2)}+C_{n}^{(n-3)}}{f_{n}}, \frac{C_{n}^{(n-2)}-3}{f_{n}},\right. \\
& \frac{C_{n}^{(1)}}{f_{n}}, \frac{C_{n}^{(2)}-3 C_{n}^{(1)}}{f_{n}}, \frac{C_{n}^{(3)}-3 C_{n}^{(2)}+C_{n}^{(1)}}{f_{n}}, \ldots, \\
& \left.\frac{C_{n}^{(n-2)}-3 C_{n}^{(n-3)}+C_{n}^{(n-4)}}{f_{n}}\right) \\
& =\frac{1}{f_{n}} \operatorname{Circ}\left(1-\sum_{i=1}^{n-2} \frac{\mathscr{F}_{n-i}\left(-\mathscr{F}_{n}\right)^{i-1}}{\left(\mathscr{F}_{1}-\mathscr{F}_{n+1}\right)^{i}},\right. \\
& -3+\sum_{i=1}^{n-2} \frac{\mathscr{F}_{n-1-i}\left(-\mathscr{F}_{n}\right)^{i-1}}{\left(\mathscr{F}_{1}-\mathscr{F}_{n+1}\right)^{i}}, \frac{1}{\mathscr{F}_{1}-\mathscr{F}_{n+1}}, \\
& \frac{-\mathscr{F}_{n}}{\left(\mathscr{F}_{1}-\mathscr{F}_{n+1}\right)^{2}}, \frac{\left(-\mathscr{F}_{n}\right)^{2}}{\left(\mathscr{F}_{1}-\mathscr{F}_{n+1}\right)^{3}}, \ldots \\
& \left.\frac{\left(-\mathscr{F}_{n}\right)^{n-3}}{\left(\mathscr{F}_{1}-\mathscr{F}_{n+1}\right)^{n-2}}\right) \text {. }
\end{aligned}
$$

Proof. Obviously, $\mathfrak{B}_{1}=2$ satisfies (31), when $n>1$. Let

$$
\begin{aligned}
& \Sigma=\left(\begin{array}{ccccccccc}
1 & & & & & & \\
-2 & & & & & & 1 \\
-1 & & & & & 1 & -1 \\
0 & & 0 & & 1 & -1 & -1 \\
\vdots & & & & . & . & . & \\
0 & & & 1 & . & . & & \\
0 & & 1 & -1 & . & & 0 & \\
0 & 1 & -1 & -1 & & & &
\end{array}\right)_{n \times n}, \\
& \Omega_{1}=\left(\begin{array}{cccccc}
1 & 0 & 0 & \cdots & 0 & 0 \\
0 & \left(\frac{\mathscr{L}_{n}-2}{\mathscr{L}_{1}-\mathscr{L}_{n+1}}\right)^{n-2} & 0 & \cdots & 0 & 1 \\
0 & \left(\frac{\mathscr{L}_{n}-2}{\mathscr{L}_{1}-\mathscr{L}_{n+1}}\right)^{n-3} & 0 & \cdots & 1 & 0 \\
\vdots & \vdots & \vdots & \ddots & \vdots & \vdots \\
0 & \frac{\mathscr{L}_{n}-2}{\mathscr{L}_{1}-\mathscr{L}_{n+1}} & 1 & \cdots & 0 & 0 \\
0 & 1 & 0 & \cdots & 0 & 0
\end{array}\right)_{n \times n}
\end{aligned}
$$

Then

$$
\begin{aligned}
& \sum \mathfrak{B}_{n} \Omega_{1} \\
& \quad\left(\begin{array}{cccccc}
\mathscr{L}_{1} & l_{n}^{\prime} & \mathscr{L}_{n-1} & \cdots & \mathscr{L}_{3} & \mathscr{L}_{2} \\
0 & l_{n}^{\prime} & -2 \mathscr{L}_{n-1}+\mathscr{L}_{n} & \cdots & \cdots & -2 \mathscr{L}_{2}+\mathscr{L}_{3} \\
0 & 0 & \mathscr{L}_{1}-\mathscr{L}_{n+1} & & & \\
0 & 0 & 2-\mathscr{L}_{n} & & & \\
\vdots & \vdots & & \ddots & & \\
0 & 0 & & & 2-\mathscr{L}_{n} & \mathscr{L}_{1}-\mathscr{L}_{n+1}
\end{array}\right),
\end{aligned}
$$

where

$$
\begin{gathered}
l_{n}=\mathscr{L}_{1}-2 \mathscr{L}_{n}+\sum_{k=1}^{n-2}\left(\mathscr{L}_{k+2}-2 \mathscr{L}_{k+1}\right)\left(\frac{\mathscr{L}_{n}-2}{\mathscr{L}_{1}-\mathscr{L}_{n+1}}\right)^{n-(k+1)} \\
l_{n}^{\prime}=\sum_{k=1}^{n-1} \mathscr{L}_{k+1}\left(\frac{\mathscr{L}_{n}-2}{\mathscr{L}_{1}-\mathscr{L}_{n+1}}\right)^{n-(k+1)}
\end{gathered}
$$

We can obtain

$\operatorname{det} \Sigma \operatorname{det} \boldsymbol{B}_{n} \operatorname{det} \Omega_{1}$

$$
\begin{aligned}
& =\mathscr{L}_{1}\left[\mathscr{L}_{1}-2 \mathscr{L}_{n}\right. \\
& \left.\quad+\sum_{k=1}^{n-2}\left(\mathscr{L}_{k+2}-2 \mathscr{L}_{k+1}\right)\left(\frac{\mathscr{L}_{n}-2}{\mathscr{L}_{1}-\mathscr{L}_{n+1}}\right)^{n-(k+1)}\right] \\
& \quad \times\left(\mathscr{L}_{1}-\mathscr{L}_{n+1}\right)^{n-2}
\end{aligned}
$$

where $\mathscr{L}_{n}$ is the nth $F_{n}+L_{n}$ number. 


$$
\begin{aligned}
& =\mathscr{L}_{1}\left[\mathscr{L}_{1}-\mathscr{L}_{n+1}\right. \\
& \left.\quad+\sum_{k=1}^{n-1}\left(\mathscr{L}_{k+2}-2 \mathscr{L}_{k+1}\right)\left(\frac{\mathscr{L}_{n}-2}{\mathscr{L}_{1}-\mathscr{L}_{n+1}}\right)^{n-(k+1)}\right] \\
& \quad \times\left(\mathscr{L}_{1}-\mathscr{L}_{n+1}\right)^{n-2} \\
& =2\left[\left(2-\mathscr{L}_{n+1}\right)^{n-1}+\left(\mathscr{L}_{n}-2\right)^{n-2}\right. \\
& \left.\quad \times \sum_{k=1}^{n-1}\left(\mathscr{L}_{k+2}-2 \mathscr{L}_{k+1}\right)\left(\frac{2-\mathscr{L}_{n+1}}{\mathscr{L}_{n}-2}\right)^{k-1}\right]
\end{aligned}
$$

while

$$
\operatorname{det} \Sigma=\operatorname{det} \Omega_{1}=(-1)^{(n-1)(n-2) / 2} .
$$

We have

$$
\begin{aligned}
\operatorname{det} & \mathfrak{B}_{n} \\
=2[ & \left(2-\mathscr{L}_{n+1}\right)^{n-1}+\left(\mathscr{L}_{n}-2\right)^{n-2} \\
& \left.\times \sum_{k=1}^{n-1}\left(\mathscr{L}_{k+2}-2 \mathscr{L}_{k+1}\right)\left(\frac{2-\mathscr{L}_{n+1}}{\mathscr{L}_{n}-2}\right)^{k-1}\right] .
\end{aligned}
$$

Theorem 13. Let $\mathfrak{B}_{n}=\operatorname{Circ}\left(\mathscr{L}_{1}, \mathscr{L}_{2}, \ldots, \mathscr{L}_{n}\right)$ be a circulant matrix; then $\mathfrak{B}_{n}$ is invertible for any positive integer $n$.

Proof. Since $\mathscr{L}_{n}=\left(\alpha^{n}-\beta^{n}\right) /(\alpha-\beta)+\alpha^{n}+\beta^{n}$, where $\alpha+\beta=1$, $\alpha \cdot \beta=-1$. We have

$$
\begin{aligned}
f\left(\omega^{k}\right)= & \sum_{j=1}^{n} \mathscr{L}_{j}\left(\omega^{k}\right)^{j-1} \\
= & \sum_{j=1}^{n}\left(\frac{\alpha^{j}-\beta^{j}}{\alpha-\beta}+\alpha^{j}+\beta^{j}\right)\left(\omega^{k}\right)^{j-1} \\
= & \sum_{j=1}^{n}\left(\frac{\alpha^{j}-\beta^{j}}{\alpha-\beta}\right)\left(\omega^{k}\right)^{j-1}+\sum_{j=1}^{n}\left(\alpha^{j}+\beta^{j}\right)\left(\omega^{k}\right)^{j-1} \\
= & \frac{1}{\alpha-\beta}\left[\frac{\alpha\left(1-\alpha^{n}\right)}{1-\alpha \omega^{k}}-\frac{\beta\left(1-\beta^{n}\right)}{1-\beta \omega^{k}}\right] \\
& +\frac{\alpha\left(1-\alpha^{n}\right)}{1-\alpha \omega^{k}}+\frac{\beta\left(1-\beta^{n}\right)}{1-\beta \omega^{k}} \\
= & \frac{1-F_{n+1}-F_{n} \omega^{k}}{1-\omega^{k}-\omega^{2 k}}+\frac{1-L_{n+1}+\left(2-L_{n}\right) \omega^{k}}{1-\omega^{k}-\omega^{2 k}}
\end{aligned}
$$

$$
\begin{aligned}
& =\frac{2-\left(F_{n+1}+L_{n+1}\right)-\left(F_{n}+L_{n}-2\right) \omega^{k}}{1-\omega^{k}-\omega^{2 k}} \\
& =\frac{2-\mathscr{L}_{n+1}-\left(\mathscr{L}_{n}-2\right) \omega^{k}}{1-\omega^{k}-\omega^{2 k}} \quad(k=1,2, \ldots, n-1) .
\end{aligned}
$$

If there exist $\omega^{l}(l=1,2, \ldots, n-1)$ such that $f\left(\omega^{l}\right)=0$, we obtain $2-\mathscr{L}_{n+1}-\left(\mathscr{L}_{n}-2\right) \omega^{l}=0$ for $1-\omega^{l}-\omega^{2 l} \neq 0 ; \omega^{l}=$ $\left(2-\mathscr{L}_{n+1}\right) /\left(\mathscr{L}_{n}-2\right)$ is a real number, while $\omega^{l}=\exp (2 l \pi i / n)=$ $\cos (2 l \pi / n)+i \sin (2 l \pi / n)$.

Hence, $\sin (2 l \pi / n)=0$, so we have $\omega^{l}=-1$ for $0<$ $2 l \pi / n<2 \pi$. But $x=-1$ is not the root of the equation $2-\mathscr{L}_{n+1}-\left(\mathscr{L}_{n}-2\right) x=0$ for any positive integer $n$. We obtain $f\left(\omega^{k}\right) \neq 0$ for any $\omega^{k}(k=1,2, \ldots, n-1)$, while $f(1)=$ $\sum_{j=1}^{n} \mathscr{L}_{j}=\mathscr{L}_{n+1}+\mathscr{L}_{n}-4 \neq 0$. By Lemma 4 , the proof is completed.

Lemma 14. Let matrix $\mathscr{H}=\left[h_{i j}\right]_{i, j=1}^{n-2}$ be of the form

$$
h_{i j}= \begin{cases}\mathscr{L}_{1}-\mathscr{L}_{n+1}, & i=j, \\ 2-\mathscr{L}_{n}, & i=j+1, \\ 0, & \text { otherwise }\end{cases}
$$

and then inverse $\mathscr{H}^{-1}=\left[h_{i, j}^{\prime}\right]_{i, j=1}^{n-2}$ of the matrix $\mathscr{H}$ is equal to

$$
h_{i j}^{\prime}= \begin{cases}\frac{\left(\mathscr{L}_{n}-2\right)^{i-j}}{\left(\mathscr{L}_{1}-\mathscr{L}_{n+1}\right)^{i-j+1}}, & i \geq j, \\ 0, & i<j .\end{cases}
$$

Proof. Let $r_{i j}=\sum_{k=1}^{n-2} h_{i k} h_{k j}^{\prime}$. Obviously, $r_{i j}=0$ for $i<j$. In the case $i=j$, we obtain

$$
r_{i i}=h_{i i} h_{i i}^{\prime}=\left(\mathscr{L}_{1}-\mathscr{L}_{n+1}\right) \cdot \frac{1}{\mathscr{L}_{1}-\mathscr{L}_{n+1}}=1 .
$$

For $i \geq j+1$, we obtain

$$
\begin{aligned}
r_{i j}= & \sum_{k=1}^{n-2} h_{i k} h_{k j}^{\prime}=h_{i, i-1} h_{i-1, j}^{\prime}+h_{i i} h_{i j}^{\prime} \\
= & \left(2-\mathscr{L}_{n}\right) \cdot \frac{\left(\mathscr{L}_{n}-2\right)^{i-j-1}}{\left(\mathscr{L}_{1}-\mathscr{L}_{n+1}\right)^{i-j}} \\
& +\left(\mathscr{L}_{1}-\mathscr{L}_{n+1}\right) \cdot \frac{\left(\mathscr{L}_{n}-2\right)^{i-j}}{\left(\mathscr{L}_{1}-\mathscr{L}_{n+1}\right)^{i-j+1}} \\
= & 0 .
\end{aligned}
$$

We verify $\mathscr{H}^{-1}=I_{n-2}$, where $I_{n-2}$ is the $(n-2) \times(n-2)$ identity matrix. Similarly, we can verify $\mathscr{H}^{-1} \mathscr{H}=I_{n-2}$. Thus, the proof is completed. 
Theorem 15. Let $\mathfrak{B}_{n}=\operatorname{Circ}\left(\mathscr{L}_{1}, \mathscr{L}_{2}, \ldots, \mathscr{L}_{n}\right)$ be a circulant matrix; then one has

$$
\begin{aligned}
\mathfrak{B}_{n}^{-1}=\frac{1}{l_{n}} \operatorname{Circ}( & 1-\sum_{i=1}^{n-2} \frac{\mathscr{L}_{n-i-1}\left(\mathscr{L}_{n}-2\right)^{i-1}}{\left(\mathscr{L}_{1}-\mathscr{L}_{n+1}\right)^{i}}, \\
& -2-\sum_{i=1}^{n-2} \frac{\left(2 \mathscr{L}_{n-i}-\mathscr{L}_{n-i+1}\right)\left(\mathscr{L}_{n}-2\right)^{i-1}}{\left(\mathscr{L}_{1}-\mathscr{L}_{n+1}\right)^{i}}, \\
& \frac{2}{\mathscr{L}_{1}-\mathscr{L}_{n+1}}, \frac{2\left(\mathscr{L}_{n}-2\right)}{\left(\mathscr{L}_{1}-\mathscr{L}_{n+1}\right)^{2}}, \\
& \left.\frac{2\left(\mathscr{L}_{n}-2\right)^{2}}{\left(\mathscr{L}_{1}-\mathscr{L}_{n+1}\right)^{3}}, \ldots, \frac{2\left(\mathscr{L}_{n}-2\right)^{n-3}}{\left(\mathscr{L}_{1}-\mathscr{L}_{n+1}\right)^{n-2}}\right),
\end{aligned}
$$

where

$$
\begin{aligned}
l_{n}= & \mathscr{L}_{1}-2 \mathscr{L}_{n} \\
& +\sum_{k=1}^{n-2}\left(\mathscr{L}_{k+2}-2 \mathscr{L}_{k+1}\right)\left(\frac{\mathscr{L}_{n}-2}{\mathscr{L}_{1}-\mathscr{L}_{n+1}}\right)^{n-(k+1)} .
\end{aligned}
$$

Proof. Let

$$
\Omega_{2}=\left(\begin{array}{cccccc}
1 & -\frac{l_{n}^{\prime}}{2} & \omega_{13} & \omega_{14} & \cdots & \omega_{1 n} \\
0 & 1 & \omega_{23} & \omega_{24} & \cdots & \omega_{2 n} \\
0 & 0 & 1 & 0 & \cdots & 0 \\
0 & 0 & 0 & 1 & \cdots & 0 \\
\vdots & \vdots & \vdots & \vdots & \ddots & \vdots \\
0 & 0 & 0 & 0 & \cdots & 1
\end{array}\right),
$$

where

$$
\begin{aligned}
\omega_{1 i}= & \frac{1}{2}\left[\frac{l_{n}^{\prime}\left(\mathscr{L}_{n+3-i}-2 \mathscr{L}_{n+2-i}\right)}{l_{n}}-\mathscr{L}_{n+2-i}\right], \\
\omega_{2 i}= & \frac{2 \mathscr{L}_{n+2-i}-\mathscr{L}_{n+3-i}}{l_{n}}, \quad i=3,4, \ldots, n, \\
l_{n}= & \mathscr{L}_{1}-2 \mathscr{L}_{n} \\
& +\sum_{k=1}^{n-2}\left(\mathscr{L}_{k+2}-2 \mathscr{L}_{k+1}\right)\left(\frac{\mathscr{L}_{n}-2}{\mathscr{L}_{1}-\mathscr{L}_{n+1}}\right)^{n-(k+1)}, \\
l_{n}^{\prime}= & \sum_{k=1}^{n-1} \mathscr{L}_{k+1}\left(\frac{\mathscr{L}_{n}-2}{\mathscr{L}_{1}-\mathscr{L}_{n+1}}\right)^{n-(k+1)} .
\end{aligned}
$$

We have

$$
\sum \mathfrak{B}_{n} \Omega_{1} \Omega_{2}=\mathscr{D}_{2} \oplus \mathscr{H}
$$

where $\mathscr{D}_{2}=\operatorname{diag}\left(\mathscr{L}_{1}, l_{n}\right)$ is a diagonal matrix and $\mathscr{D}_{2} \oplus \mathscr{H}$ is the direct sum of $\mathscr{D}_{2}$ and $\mathscr{H}$. If we denote $\Omega=\Omega_{1} \Omega_{2}$, then we obtain

$$
\mathfrak{B}_{n}^{-1}=\Omega\left(\mathscr{D}_{2}^{-1} \oplus \mathscr{H}^{-1}\right) \Sigma
$$

Since the last row elements of the matrix $\Omega$ are

$$
0,1, \frac{2 \mathscr{L}_{n-1}-\mathscr{L}_{n}}{l_{n}}, \frac{2 \mathscr{L}_{n-2}-\mathscr{L}_{n-1}}{l_{n}}, \ldots, \frac{2 \mathscr{L}_{2}-\mathscr{L}_{3}}{l_{n}}
$$

By Lemma 14, if we let $\mathfrak{B}_{n}^{-1}=\operatorname{Circ}\left(y_{1}, y_{2}, \ldots, y_{n}\right)$, then its last row elements are given by the following equations:

$$
\begin{aligned}
& y_{2}=-\frac{2}{l_{n}}-\frac{1}{l_{n}} \sum_{i=1}^{n-2} \frac{\left(2 \mathscr{L}_{n-i}-\mathscr{L}_{n-i+1}\right)\left(\mathscr{L}_{n}-2\right)^{i-1}}{\left(\mathscr{L}_{1}-\mathscr{L}_{n+1}\right)^{i}} \text {, } \\
& y_{3}=\frac{1}{l_{n}} \frac{2 \mathscr{L}_{2}-\mathscr{L}_{3}}{\left(\mathscr{L}_{1}-\mathscr{L}_{n+1}\right)} \text {, } \\
& y_{4}=-\frac{1}{l_{n}} \frac{2 \mathscr{L}_{2}-\mathscr{L}_{3}}{\left(\mathscr{L}_{1}-\mathscr{L}_{n+1}\right)} \\
& +\frac{1}{l_{n}} \sum_{i=1}^{2} \frac{\left(2 \mathscr{L}_{4-i}-\mathscr{L}_{5-i}\right)\left(\mathscr{L}_{n}-2\right)^{i-1}}{\left(\mathscr{L}_{1}-\mathscr{L}_{n+1}\right)^{i}} \\
& y_{5}=-\frac{1}{l_{n}} \frac{2 \mathscr{L}_{2}-\mathscr{L}_{3}}{\left(\mathscr{L}_{1}-\mathscr{L}_{n+1}\right)} \\
& -\frac{1}{l_{n}} \sum_{i=1}^{2} \frac{\left(2 \mathscr{L}_{4-i}-\mathscr{L}_{5-i}\right)\left(\mathscr{L}_{n}-2\right)^{i-1}}{\left(\mathscr{L}_{1}-\mathscr{L}_{n+1}\right)^{i}} \\
& +\frac{1}{l_{n}} \sum_{i=1}^{3} \frac{\left(2 \mathscr{L}_{5-i}-\mathscr{L}_{6-i}\right)\left(\mathscr{L}_{n}-2\right)^{i-1}}{\left(\mathscr{L}_{1}-\mathscr{L}_{n+1}\right)^{i}} \\
& y_{n}=\frac{1}{l_{n}} \sum_{i=1}^{n-2} \frac{\left(2 \mathscr{L}_{n-i}-\mathscr{L}_{n-i+1}\right)\left(\mathscr{L}_{n}-2\right)^{i-1}}{\left(\mathscr{L}_{1}-\mathscr{L}_{n+1}\right)^{i}} \\
& -\frac{1}{l_{n}} \sum_{i=1}^{n-3} \frac{\left(2 \mathscr{L}_{n-i-1}-\mathscr{L}_{n-i}\right)\left(\mathscr{L}_{n}-2\right)^{i-1}}{\left(\mathscr{L}_{1}-\mathscr{L}_{n+1}\right)^{i}} \\
& -\frac{1}{l_{n}} \sum_{i=1}^{n-4} \frac{\left(2 \mathscr{L}_{n-i-2}-\mathscr{L}_{n-i-1}\right)\left(\mathscr{L}_{n}-2\right)^{i-1}}{\left(\mathscr{L}_{1}-\mathscr{L}_{n+1}\right)^{i}} \text {, } \\
& y_{1}=\frac{1}{l_{n}}\left[1-\sum_{i=1}^{n-2} \frac{\left(2 \mathscr{L}_{n-i}-\mathscr{L}_{n-i+1}\right)\left(\mathscr{L}_{n}-2\right)^{i-1}}{\left(\mathscr{L}_{1}-\mathscr{L}_{n+1}\right)^{i}}\right. \\
& \left.-\sum_{i=1}^{n-3} \frac{\left(2 \mathscr{L}_{n-i-1}-\mathscr{L}_{n-i}\right)\left(\mathscr{L}_{n}-2\right)^{i-1}}{\left(\mathscr{L}_{1}-\mathscr{L}_{n+1}\right)^{i}}\right] \text {. }
\end{aligned}
$$

Let $D_{n}^{(j)}=\sum_{i=1}^{j}\left(\left(2 \mathscr{L}_{j+2-i}-\mathscr{L}_{j+3-i}\right)\left(\mathscr{L}_{n}-2\right)^{i-1} /\right.$ $\left.\left(\mathscr{L}_{1}-\mathscr{L}_{n+1}\right)^{i}\right)(j=1,2, \ldots, n-2)$; we have

$$
\begin{aligned}
D_{n}^{(2)}- & D_{n}^{(1)} \\
= & -\frac{2 \mathscr{L}_{2}-\mathscr{L}_{3}}{\mathscr{L}_{1}-\mathscr{L}_{n+1}}+\sum_{i=1}^{2} \frac{\left(2 \mathscr{L}_{4-i}-\mathscr{L}_{5-i}\right)\left(\mathscr{L}_{n}-2\right)^{i-1}}{\left(\mathscr{L}_{1}-\mathscr{L}_{n+1}\right)^{i}}
\end{aligned}
$$




$$
\begin{aligned}
& =\frac{2\left(\mathscr{L}_{n}-2\right)}{\left(\mathscr{L}_{1}-\mathscr{L}_{n+1}\right)^{2}} \\
& D_{n}^{(n-3)}+D_{n}^{(n-2)} \\
& =\sum_{i=1}^{n-3} \frac{\left(2 \mathscr{L}_{n-i-1}-\mathscr{L}_{n-i}\right)\left(\mathscr{L}_{n}-2\right)^{i-1}}{\left(\mathscr{L}_{1}-\mathscr{L}_{n+1}\right)^{i}} \\
& +\sum_{i=1}^{n-2} \frac{\left(2 \mathscr{L}_{n-i}-\mathscr{L}_{n-i+1}\right)\left(\mathscr{L}_{n}-2\right)^{i-1}}{\left(\mathscr{L}_{1}-\mathscr{L}_{n+1}\right)^{i}} \\
& =\frac{\left(2 \mathscr{L}_{2}-\mathscr{L}_{3}\right)\left(\mathscr{L}_{n}-2\right)^{n-3}}{\left(\mathscr{L}_{1}-\mathscr{L}_{n+1}\right)^{n-2}}+\sum_{i=1}^{n-3} \frac{\mathscr{L}_{n-i-1}\left(\mathscr{L}_{n}-2\right)^{i-1}}{\left(\mathscr{L}_{1}-\mathscr{L}_{n+1}\right)^{i}} \\
& =\sum_{i=1}^{n-2} \frac{\mathscr{L}_{n-i-1}\left(\mathscr{L}_{n}-2\right)^{i-1}}{\left(\mathscr{L}_{1}-\mathscr{L}_{n+1}\right)^{i}} \\
& D_{n}^{(j+2)}-D_{n}^{(j+1)}-D_{n}^{(j)} \\
& =\sum_{i=1}^{j+2} \frac{\left(2 \mathscr{L}_{j-i+4}-\mathscr{L}_{j-i+5}\right)\left(\mathscr{L}_{n}-2\right)^{i-1}}{\left(\mathscr{L}_{1}-\mathscr{L}_{n+1}\right)^{i}} \\
& -\sum_{i=1}^{j+1} \frac{\left(2 \mathscr{L}_{j-i+3}-\mathscr{L}_{j-i+4}\right)\left(\mathscr{L}_{n}-2\right)^{i-1}}{\left(\mathscr{L}_{1}-\mathscr{L}_{n+1}\right)^{i}} \\
& -\sum_{i=1}^{j} \frac{\left(2 \mathscr{L}_{j-i+2}-\mathscr{L}_{j-i+3}\right)\left(\mathscr{L}_{n}-2\right)^{i-1}}{\left(\mathscr{L}_{1}-\mathscr{L}_{n+1}\right)^{i}} \\
& =\frac{\left(2 \mathscr{L}_{2}-\mathscr{L}_{3}\right)\left(\mathscr{L}_{n}-2\right)^{j+1}}{\left(\mathscr{L}_{1}-\mathscr{L}_{n+1}\right)^{j+2}} \\
& +\frac{\left(2 \mathscr{L}_{3}-\mathscr{L}_{4}\right)\left(\mathscr{L}_{n}-2\right)^{j}}{\left(\mathscr{L}_{1}-\mathscr{L}_{n+1}\right)^{j+1}}-\frac{\left(2 \mathscr{L}_{2}-\mathscr{L}_{3}\right)\left(\mathscr{L}_{n}-2\right)^{j}}{\left(\mathscr{L}_{1}-\mathscr{L}_{n+1}\right)^{j+1}} \\
& =\frac{2\left(\mathscr{L}_{n}-2\right)^{j+1}}{\left(\mathscr{L}_{1}-\mathscr{L}_{n+1}\right)^{j+2}} \quad(j=1,2, \ldots, n-4) .
\end{aligned}
$$

We obtain

$\mathfrak{B}_{n}^{-1}$

$$
\begin{aligned}
=\operatorname{Circ}( & \frac{1-D_{n}^{(n-2)}-D_{n}^{(n-3)}}{l_{n}}, \frac{-2-D_{n}^{(n-2)}}{l_{n}}, \\
& \frac{D_{n}^{(1)}}{l_{n}}, \frac{D_{n}^{(2)}-D_{n}^{(1)}}{l_{n}}, \frac{D_{n}^{(3)}-D_{n}^{(2)}-D_{n}^{(1)}}{l_{n}}, \ldots, \\
& \left.\frac{D_{n}^{(n-2)}-D_{n}^{(n-3)}-D_{n}^{(n-4)}}{l_{n}}\right)
\end{aligned}
$$

$$
\begin{aligned}
=\frac{1}{l_{n}} \operatorname{Circ}( & 1-\sum_{i=1}^{n-2} \frac{\mathscr{L}_{n-i-1}\left(\mathscr{L}_{n}-2\right)^{i-1}}{\left(\mathscr{L}_{1}-\mathscr{L}_{n+1}\right)^{i}}, \\
& -2-\sum_{i=1}^{n-2} \frac{\left(2 \mathscr{L}_{n-i}-\mathscr{L}_{n-i+1}\right)\left(\mathscr{L}_{n}-2\right)^{i-1}}{\left(\mathscr{L}_{1}-\mathscr{L}_{n+1}\right)^{i}}, \\
& \frac{2}{\mathscr{L}_{1}-\mathscr{L}_{n+1}}, \frac{2\left(\mathscr{L}_{n}-2\right)}{\left(\mathscr{L}_{1}-\mathscr{L}_{n+1}\right)^{2}}, \frac{2\left(\mathscr{L}_{n}-2\right)^{2}}{\left(\mathscr{L}_{1}-\mathscr{L}_{n+1}\right)^{3}}, \ldots, \\
& \left.\frac{2\left(\mathscr{L}_{n}-2\right)^{n-3}}{\left(\mathscr{L}_{1}-\mathscr{L}_{n+1}\right)^{n-2}}\right) .
\end{aligned}
$$

\section{Determinant and Inverse of a Left Circulant Matrix with $\mathscr{F}_{n}$ and $\mathscr{L}_{n}$ Numbers}

In this section, let $\mathscr{A}_{n}^{\prime}=\operatorname{LCirc}\left(\mathscr{F}_{1}, \mathscr{F}_{2}, \ldots, \mathscr{F}_{n}\right)$ and $\mathfrak{B}_{n}^{\prime}=$ LCirc $\left(\mathfrak{Q}_{1}, \mathfrak{\Omega}_{2}, \ldots, \mathbb{R}_{n}\right)$ be left circulant matrices. By using the obtained conclusions, we give a determinant formula for the matrix $\mathscr{A}_{n}^{\prime}$ and $\mathfrak{B}_{n}^{\prime}$. Afterwards, we prove that $\mathscr{A}_{n}^{\prime}$ is an invertible matrix for $n>2$ and $\mathfrak{B}_{n}^{\prime}$ is an invertible matrix for any positive integer $n$. The inverses of the matrices $\mathscr{A}_{n}^{\prime}$ and $\mathfrak{B}_{n}^{\prime}$ are also presented.

According to Lemma 5 and Theorems 8, 9, and 11, we can obtain the following theorems.

Theorem 16. Let $\mathscr{A}_{n}^{\prime}=\operatorname{LCirc}\left(\mathscr{F}_{1}, \mathscr{F}_{2}, \ldots, \mathscr{F}_{n}\right)$ be a left circulant matrix; then one has

$$
\begin{aligned}
\operatorname{det} \mathscr{A}_{n}^{\prime}=( & -1)^{(n-1)(n-2) / 2} \\
\times & {\left[\left(1+\mathscr{F}_{n+1}\right)^{n-1}\right.} \\
& \left.+\left(-\mathscr{F}_{n}\right)^{n-2} \sum_{k=1}^{n-1}\left(-\mathscr{F}_{k}\right)\left(\frac{1+\mathscr{F}_{n+1}}{-\mathscr{F}_{n}}\right)^{k-1}\right],
\end{aligned}
$$

where $\mathscr{F}_{n}$ is the nth $F_{n} \cdot L_{n}$ number.

Theorem 17. Let $\mathscr{A}_{n}^{\prime}=\operatorname{LCirc}\left(\mathscr{F}_{1}, \mathscr{F}_{2}, \ldots, \mathscr{F}_{n}\right)$ be a left circulant matrix; if $n>2$, then $\mathscr{A}_{n}^{\prime}$ is an invertible matrix.

Theorem 18. Let $\mathscr{A}_{n}^{\prime}=\operatorname{LCirc}\left(\mathscr{F}_{1}, \mathscr{F}_{2}, \ldots, \mathscr{F}_{n}\right)(n>2)$ be a left circulant matrix; then one has

$$
\begin{aligned}
\mathscr{A}_{n}^{\prime-1}=\frac{1}{f_{n}} \operatorname{LCirc}\left(1-\sum_{i=1}^{n-2} \frac{\mathscr{F}_{n-i}\left(-\mathscr{F}_{n}\right)^{i-1}}{\left(\mathscr{F}_{1}-\mathscr{F}_{n+1}\right)^{i}},\right. \\
\\
\frac{\left(-\mathscr{F}_{n}\right)^{n-3}}{\left(\mathscr{F}_{1}-\mathscr{F}_{n+1}\right)^{n-2}}, \ldots, \frac{\left(-\mathscr{F}_{n}\right)^{2}}{\left(\mathscr{F}_{1}-\mathscr{F}_{n+1}\right)^{3}},
\end{aligned}
$$




$$
\begin{aligned}
& \frac{-\mathscr{F}_{n}}{\left(\mathscr{F}_{1}-\mathscr{F}_{n+1}\right)^{2}}, \frac{1}{\mathscr{F}_{1}-\mathscr{F}_{n+1}}, \\
& \left.-3+\sum_{i=1}^{n-2} \frac{\mathscr{F}_{n-1-i}\left(-\mathscr{F}_{n}\right)^{i-1}}{\left(\mathscr{F}_{1}-\mathscr{F}_{n+1}\right)^{i}}\right),
\end{aligned}
$$

where

$$
f_{n}=\mathscr{F}_{1}-3 \mathscr{F}_{n}+\sum_{k=1}^{n-2}\left(-\mathscr{F}_{k}\right)\left(\frac{-\mathscr{F}_{n}}{\mathscr{F}_{1}-\mathscr{F}_{n+1}}\right)^{n-(k+1)} .
$$

By Lemma 5 and Theorems 12, 13, and 15, the following conclusions can be attained.

Theorem 19. Let $\mathfrak{B}_{n}^{\prime}=\operatorname{LCirc}\left(\mathscr{L}_{1}, \mathscr{L}_{2}, \ldots, \mathscr{L}_{n}\right)$ be a left circulant matrix; then one has

$$
\begin{aligned}
\operatorname{det} \mathfrak{B}_{n}^{\prime}= & 2(-1)^{(n-1)(n-2) / 2} \\
& \times\left[\left(2-\mathscr{L}_{n+1}\right)^{n-1}+\left(\mathscr{L}_{n}-2\right)^{n-2}\right. \\
& \left.\quad \times \sum_{k=1}^{n-1}\left(\mathscr{L}_{k+2}-2 \mathscr{L}_{k+1}\right)\left(\frac{2-\mathscr{L}_{n+1}}{\mathscr{L}_{n}-2}\right)^{k-1}\right],
\end{aligned}
$$

where $\mathscr{L}_{n}$ is the nth $F_{n}+L_{n}$ number.

Theorem 20. Let $\mathfrak{B}_{n}^{\prime}=\operatorname{LCirc}\left(\mathscr{L}_{1}, \mathscr{L}_{2}, \ldots, \mathscr{L}_{n}\right)$ be a left circulant matrix; then $\mathfrak{B}_{n}^{\prime}$ is invertible for any positive integer $n$.

Theorem 21. Let $\mathfrak{B}_{n}^{\prime}=\operatorname{LCirc}\left(\mathscr{L}_{1}, \mathscr{L}_{2}, \ldots, \mathscr{L}_{n}\right)$ be a left circulant matrix; then one can obtain

$$
\begin{aligned}
\mathfrak{B}_{n}^{\prime-1} & \frac{1}{l_{n}} L \operatorname{Circ}\left(1-\sum_{i=1}^{n-2} \frac{\mathscr{L}_{n-i-1}\left(\mathscr{L}_{n}-2\right)^{i-1}}{\left(\mathscr{L}_{1}-\mathscr{L}_{n+1}\right)^{i}},\right. \\
& \frac{2\left(\mathscr{L}_{n}-2\right)^{n-3}}{\left(\mathscr{L}_{1}-\mathscr{L}_{n+1}\right)^{n-2}}, \ldots, \frac{2\left(\mathscr{L}_{n}-2\right)^{2}}{\left(\mathscr{L}_{1}-\mathscr{L}_{n+1}\right)^{3}}, \\
& \frac{2\left(\mathscr{L}_{n}-2\right)}{\left(\mathscr{L}_{1}-\mathscr{L}_{n+1}\right)^{2}}, \frac{2}{\mathscr{L}_{1}-\mathscr{L}_{n+1}}, \\
& \left.-2-\sum_{i=1}^{n-2} \frac{\left(2 \mathscr{L}_{n-i}-\mathscr{L}_{n-i+1}\right)\left(\mathscr{L}_{n}-2\right)^{i-1}}{\left(\mathscr{L}_{1}-\mathscr{L}_{n+1}\right)^{i}}\right),
\end{aligned}
$$

where

$$
\begin{aligned}
l_{n}= & \mathscr{L}_{1}-2 \mathscr{L}_{n} \\
& +\sum_{k=1}^{n-2}\left(\mathscr{L}_{k+2}-2 \mathscr{L}_{k+1}\right)\left(\frac{\mathscr{L}_{n}-2}{\mathscr{L}_{1}-\mathscr{L}_{n+1}}\right)^{n-(k+1)} .
\end{aligned}
$$

\section{Determinant and Inverse of $g$-Circulant Matrix with $\mathscr{F}_{n}$ and $\mathscr{L}_{n}$ Numbers}

In this section, let $\mathscr{A}_{g, n}=g-\operatorname{Circ}\left(\mathscr{F}_{1}, \mathscr{F}_{2}, \ldots, \mathscr{F}_{n}\right)$ and $\mathfrak{B}_{g, n}=g$-Circ $\left(\mathscr{L}_{1}, \mathscr{L}_{2}, \ldots, \mathscr{L}_{n}\right)$ be $g$-circulant matrices. By using the obtained conclusions, we give a determinant formula for the matrices $\mathscr{A}_{g, n}$ and $\mathfrak{B}_{g, n}$. Afterwards, we prove that $\mathscr{A}_{g, n}$ is an invertible matrix for $n>2$ and $\mathfrak{B}_{g, n}$ is an invertible matrix if $(n, g)=1$. The inverse of the matrices $\mathscr{A}_{g, n}$ and $\mathfrak{B}_{g, n}$ are also presented.

From Lemmas 6 and 7 and Theorems 8, 9, and 11, we deduce the following results.

Theorem 22. Let $\mathscr{A}_{g, n}=g$ - $\operatorname{Circ}\left(\mathscr{F}_{1}, \mathscr{F}_{2}, \ldots, \mathscr{F}_{n}\right)$ be a g-circulant matrix; then one has

$$
\begin{aligned}
\operatorname{det} \mathscr{A}_{g, n}=\operatorname{det} \mathbb{Q}_{g}[ & \left(1+\mathscr{F}_{n+1}\right)^{n-1}+\left(-\mathscr{F}_{n}\right)^{n-2} \\
& \left.\times \sum_{k=1}^{n-1}\left(-\mathscr{F}_{k}\right)\left(\frac{1+\mathscr{F}_{n+1}}{-\mathscr{F}_{n}}\right)^{k-1}\right],
\end{aligned}
$$

where $\mathscr{F}_{n}$ is the $n$th $F_{n} \cdot L_{n}$ number.

Theorem 23. Let $\mathscr{A}_{g, n}=g$ - $\operatorname{Circ}\left(\mathscr{F}_{1}, \mathscr{F}_{2}, \ldots, \mathscr{F}_{n}\right)$ be a g-circulant matrix and $(g, n)=1 ;$ if $n>2$, then $\mathscr{A}_{g, n}$ is an invertible matrix.

Theorem 24. Let $\mathscr{A}_{g, n}=g-\operatorname{Circ}\left(\mathscr{F}_{1}, \mathscr{F}_{2}, \ldots, \mathscr{F}_{n}\right)(n>2)$ be a $g$-circulant matrix and $(g, n)=1$; then

$$
\begin{aligned}
\mathscr{A}_{g, n}^{-1} & =\left[\frac { 1 } { f _ { n } } \operatorname { C i r c } \left(1-\sum_{i=1}^{n-2} \frac{\mathscr{F}_{n-i}\left(-\mathscr{F}_{n}\right)^{i-1}}{\left(\mathscr{F}_{1}-\mathscr{F}_{n+1}\right)^{i}},\right.\right. \\
& -3+\sum_{i=1}^{n-2} \frac{\mathscr{F}_{n-1-i}\left(-\mathscr{F}_{n}\right)^{i-1}}{\left(\mathscr{F}_{1}-\mathscr{F}_{n+1}\right)^{i}}, \\
& \frac{1}{\mathscr{F}_{1}-\mathscr{F}_{n+1}}, \frac{-\mathscr{F}_{n}}{\left(\mathscr{F}_{1}-\mathscr{F}_{n+1}\right)^{2}}, \\
& \left.\left.\frac{\left(-\mathscr{F}_{n}\right)^{2}}{\left(\mathscr{F}_{1}-\mathscr{F}_{n+1}\right)^{3}}, \ldots, \frac{\left(-\mathscr{F}_{n}\right)^{n-3}}{\left(\mathscr{F}_{1}-\mathscr{F}_{n+1}\right)^{n-2}}\right)\right] \mathbb{Q}_{g}^{T},
\end{aligned}
$$

where

$$
f_{n}=\mathscr{F}_{1}-3 \mathscr{F}_{n}+\sum_{k=1}^{n-2}\left(-\mathscr{F}_{k}\right)\left(\frac{-\mathscr{F}_{n}}{\mathscr{F}_{1}-\mathscr{F}_{n+1}}\right)^{n-(k+1)} .
$$

Taking Lemmas 6 and 7 and Theorems 12,13, and 15 into account, one has the following theorems. 
Theorem 25. Let $\mathfrak{B}_{g, n}=g-\operatorname{Circ}\left(\mathscr{L}_{1}, \mathscr{L}_{2}, \ldots, \mathscr{L}_{n}\right)$ be a $g$ circulant matrix; then one has

$$
\begin{aligned}
\operatorname{det} \mathfrak{B}_{g, n}=2 \operatorname{det} & \mathbb{Q}_{g} \\
\times & {\left[\left(2-\mathscr{L}_{n+1}\right)^{n-1}+\left(\mathscr{L}_{n}-2\right)^{n-2}\right.} \\
& \left.\times \sum_{k=1}^{n-1}\left(\mathscr{L}_{k+2}-2 \mathscr{L}_{k+1}\right)\left(\frac{2-\mathscr{L}_{n+1}}{\mathscr{L}_{n}-2}\right)^{k-1}\right],
\end{aligned}
$$

where $\mathscr{L}_{n}$ is the $n F_{n}+L_{n}$ number.

Theorem 26. Let $\mathfrak{B}_{g, n}=g$-Circ $\left(\mathscr{L}_{1}, \mathscr{L}_{2}, \ldots, \mathscr{L}_{n}\right)$ be a $g$-circulant matrix and $(g, n)=1$; then $\mathfrak{B}_{g, n}$ is invertible for any positive integer $n$.

Theorem 27. Let $\mathfrak{B}_{g, n}=g$-Circ $\left(\mathscr{L}_{1}, \mathscr{L}_{2}, \ldots, \mathscr{L}_{n}\right)$ be a $g$-circulant matrix and $(g, n)=1$; then

$$
\begin{aligned}
& \mathfrak{B}_{g, n}^{-1} \\
& =\left[\frac { 1 } { l _ { n } } \operatorname { C i r c } \left(1-\sum_{i=1}^{n-2} \frac{\mathscr{L}_{n-i-1}\left(\mathscr{L}_{n}-2\right)^{i-1}}{\left(\mathscr{L}_{1}-\mathscr{L}_{n+1}\right)^{i}},\right.\right. \\
& -2-\sum_{i=1}^{n-2} \frac{\left(2 \mathscr{L}_{n-i}-\mathscr{L}_{n-i+1}\right)\left(\mathscr{L}_{n}-2\right)^{i-1}}{\left(\mathscr{L}_{1}-\mathscr{L}_{n+1}\right)^{i}}, \\
& \frac{2}{\mathscr{L}_{1}-\mathscr{L}_{n+1}}, \frac{2\left(\mathscr{L}_{n}-2\right)}{\left(\mathscr{L}_{1}-\mathscr{L}_{n+1}\right)^{2}}, \\
& \left.\left.\frac{2\left(\mathscr{L}_{n}-2\right)^{2}}{\left(\mathscr{L}_{1}-\mathscr{L}_{n+1}\right)^{3}}, \ldots, \frac{2\left(\mathscr{L}_{n}-2\right)^{n-3}}{\left(\mathscr{L}_{1}-\mathscr{L}_{n+1}\right)^{n-2}}\right)\right] \mathbb{Q}_{g}^{T} \text {, }
\end{aligned}
$$

where

$$
\begin{aligned}
l_{n}= & \mathscr{L}_{1}-2 \mathscr{L}_{n} \\
& +\sum_{k=1}^{n-2}\left(\mathscr{L}_{k+2}-2 \mathscr{L}_{k+1}\right)\left(\frac{\mathscr{L}_{n}-2}{\mathscr{L}_{1}-\mathscr{L}_{n+1}}\right)^{n-(k+1)} .
\end{aligned}
$$

\section{Conflict of Interests}

The authors declare that there is no conflict of interests regarding the publication of this paper.

\section{Acknowledgments}

The research was supported by the Development Project of Science \& Technology of Shandong Province (Grant no. 2012GGX10115) and NSFC (Grant no. 11301252) and the AMEP of Linyi University, China.

\section{References}

[1] J. C. Ruiz-Claeyssen and L. A. dos Santos Leal, "Diagonalization and spectral decomposition of factor block circulant matrices," Linear Algebra and its Applications, vol. 99, pp. 41-61, 1988.

[2] J. Wu and X. Zou, "Asymptotic and periodic boundary value problems of mixed FDEs and wave solutions of lattice differential equations," Journal of Differential Equations, vol. 135, no. 2, pp. 315-357, 1997.

[3] E. Ahmed, A. M. A. El-Sayed, and H. A. A. El-Saka, "On some Routh-Hurwitz conditions for fractional order differential equations and their applications in Lorenz, Rössler, Chua and Chen systems," Physics Letters A, vol. 358, no. 1, pp. 1-4, 2006.

[4] E. Ahmed and A. S. Elgazzar, "On fractional order differential equations model for nonlocal epidemics," Physica A: Statistical Mechanics and its Applications, vol. 379, pp. 607-614, 2007.

[5] W. F. Trench, "On nonautonomous linear systems of differential and difference equations with $R$-symmetric coefficient matrices," Linear Algebra and Its Applications, vol. 431, no. 11, pp. 2109-2117, 2009.

[6] P. E. Kloeden, A. Neuenkirch, and R. Pavani, "Multilevel Monte Carlo for stochastic differential equations with additive fractional noise," Annals of Operations Research, vol. 189, pp. 255-276, 2011.

[7] S. J. Guo, Y. M. Chen, and J. H. Wu, "Equivariant normal forms for parameterized delay differential equations with applications to bifurcation theory," Acta Mathematica Sinica, vol. 28, no. 4, pp. 825-856, 2012.

[8] F.-R. Lin and H.-X. Yang, "A fast stationary iterative method for a partial integro-differential equation in pricing options," Calcolo: A Quarterly on Numerical Analysis and Theory of Computation, vol. 50, no. 4, pp. 313-327, 2013.

[9] S. T. Lee, J. Liu, and H.-W. Sun, "Combined compact difference scheme for linear second-order partial differential equations with mixed derivative," Journal of Computational and Applied Mathematics, vol. 264, pp. 23-37, 2014.

[10] P. J. Davis, Circulant Matrices, John Wiley \& Sons, New York, NY, USA, 1979.

[11] Z. L. Jiang and Z. X. Zhou, Circulant Matrices, Chengdu Technology University Publishing Company, Chengdu, China, 1999.

[12] R. M. Gray, "Toeplitz and circulant matrices: a review," Foundations and Trends in Communication and Information Theory, vol. 2, pp. 155-239, 2006.

[13] A. Bose, R. S. Hazra, and K. Saha, "Poisson convergence of eigenvalues of circulant type matrices," Extremes: Statistical Theory and Applications in Science, Engineering and Economics, vol. 14, no. 4, pp. 365-392, 2011.

[14] A. Bose, R. S. Hazra, and K. Saha, "Spectral norm of circulanttype matrices," Journal of Theoretical Probability, vol. 24, no. 2, pp. 479-516, 2011.

[15] C. Erbas and M. M. Tanik, "Generating solutions to the $N$ queens problem using $g$-circulants," Mathematics Magazine, vol. 68, no. 5, pp. 343-356, 1995.

[16] Y.-K. Wu, R.-Z. Jia, and Q. Li, " $(0,1)$-circulant solutions to the $A^{m}=J_{n}$ matrix equation $g$," Linear Algebra and Its Applications, vol. 345, pp. 195-224, 2002.

[17] E. Ngondiep, S. Serra-Capizzano, and D. Sesana, "Spectral features and asymptotic properties for $(0,1)$-circulants and 
$(0,1)$-Toeplitz sequences,' SIAM Journal on Matrix Analysis and Applications, vol. 31, no. 4, pp. 1663-1687, 2010.

[18] A. Basak and A. Bose, "Limiting spectral distributions of some band matrices," Periodica Mathematica Hungarica, vol. 63, no. 1, pp. 113-150, 2011.

[19] A. Bose and J. Mitra, "Limiting spectral distribution of a special circulant," Statistics \& Probability Letters, vol. 60, no. 1, pp. 111$120,2002$.

[20] L. Dazheng, "Fibonacci-Lucas quasi-cyclic matrices," The Fibonacci Quarterly, vol. 40, no. 3, pp. 280-286, 2002.

[21] S.-Q. Shen, J.-M. Cen, and Y. Hao, "On the determinants and inverses of circulant matrices with Fibonacci and Lucas numbers," Applied Mathematics and Computation, vol. 217, no. 23, pp. 9790-9797, 2011.

[22] F. L. Lu and Z. L. Jiang, "The sum and product of Fibonacci and Lucas numbers, Pell and Pell-Lucas numbers representation by matrix method," WSEAS Transactions on Mathematics, vol. 12, no. 4 , p. 449.

[23] D. V. Jaiswal, "On determinants involving generalized Fibonacci numbers," The Fibonacci Quarterly, vol. 7, pp. 319-330, 1969.

[24] D. A. Lind, "A Fibonacci circulant," The Fibonacci Quarterly, vol. 8, no. 5, pp. 449-455, 1970.

[25] D. Bozkurt and T.-Y. Tam, "Determinants and inverses of circulant matrices with Jacobsthal and Jacobsthal-Lucas Numbers," Applied Mathematics and Computation, vol. 219, no. 2, pp. 544551, 2012.

[26] H. Karner, J. Schneid, and C. W. Ueberhuber, "Spectral decomposition of real circulant matrices," Linear Algebra and Its Applications, vol. 367, pp. 301-311, 2003.

[27] W. T. Stallings and T. L. Boullion, "The pseudoinverse of an $r$-circulant matrix," Proceedings of the American Mathematical Society, vol. 34, pp. 385-388, 1972. 


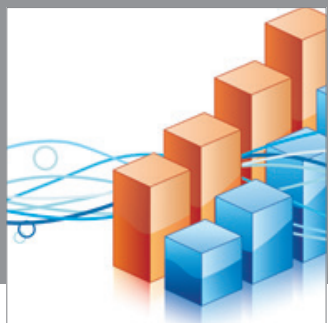

Advances in

Operations Research

mansans

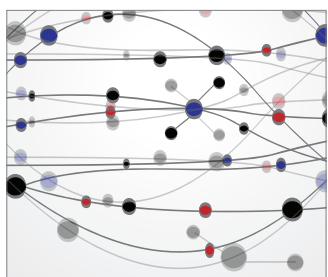

The Scientific World Journal
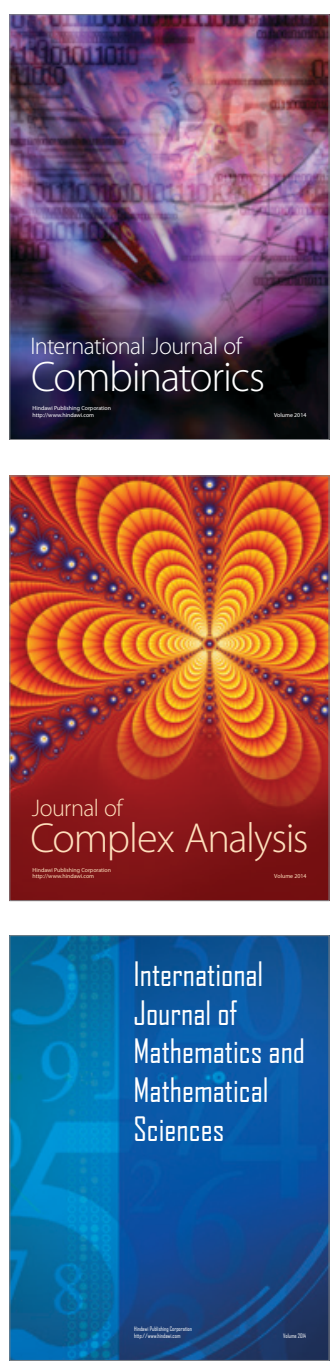
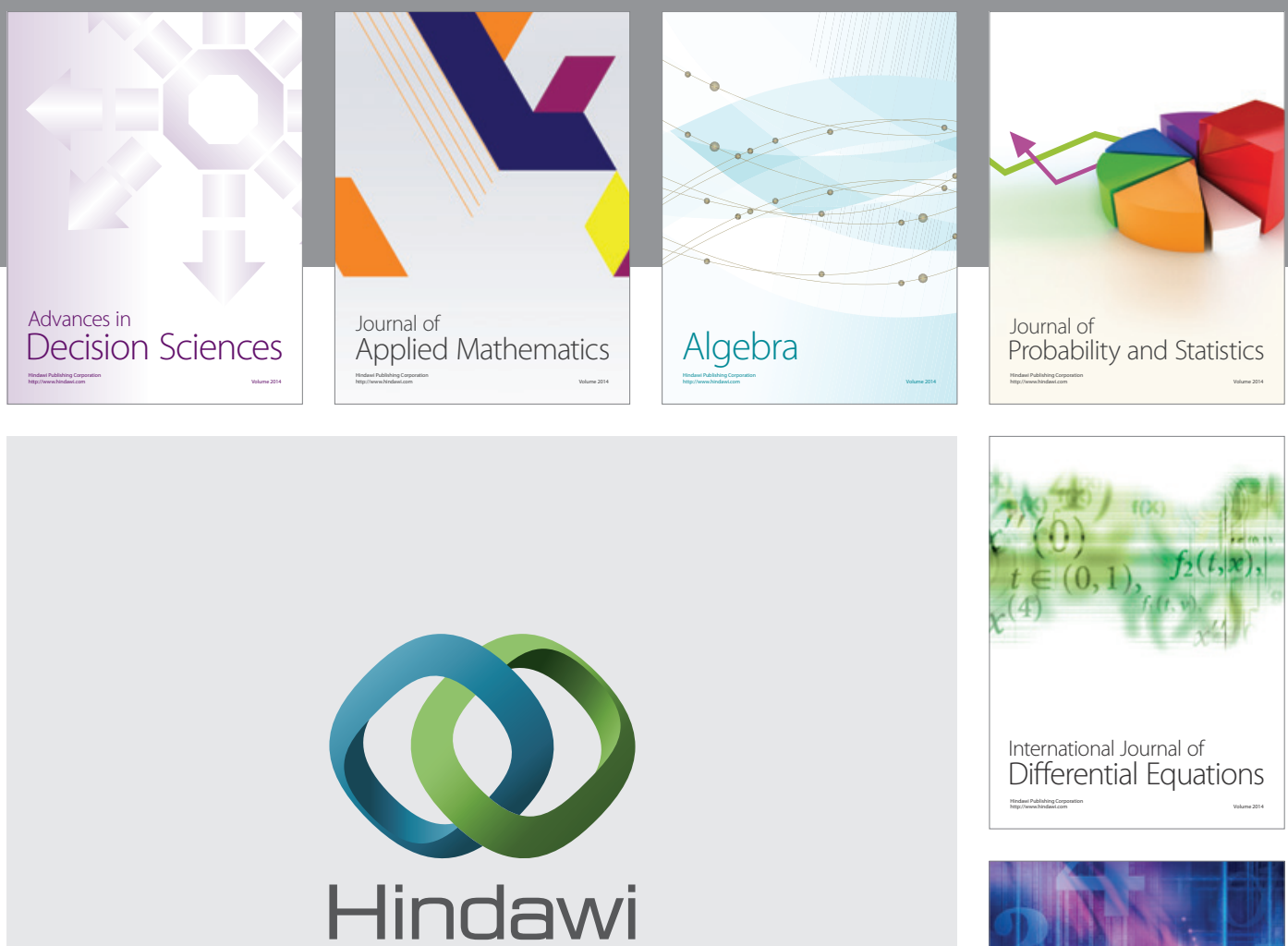

Submit your manuscripts at http://www.hindawi.com
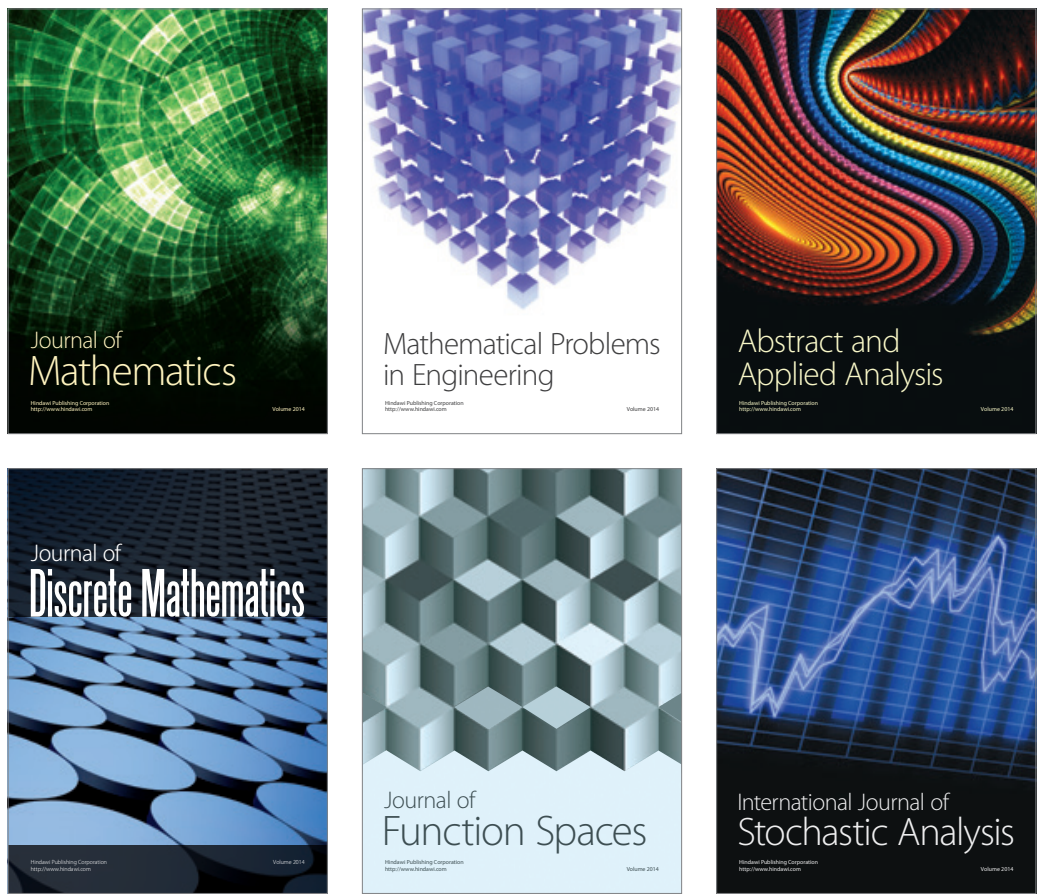

Journal of

Function Spaces

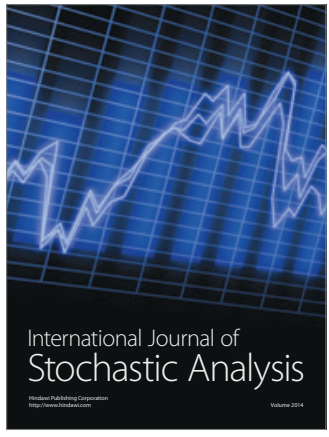

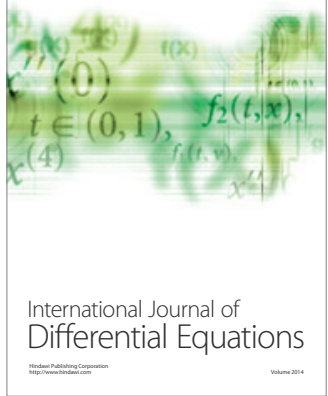
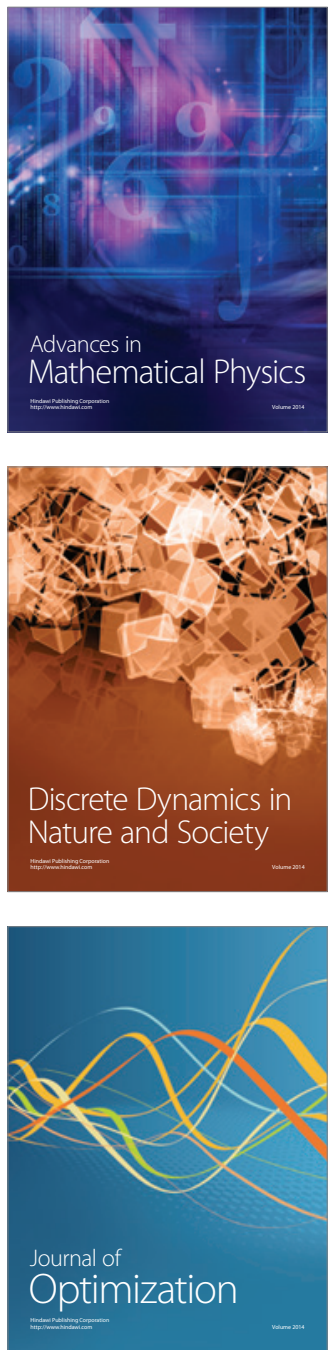